PHYSICAL REVIEW D 93, 079902(E) (2016)

\title{
Erratum: Bounds on neutrino-scalar Yukawa coupling \\ [Phys. Rev. D 93, 053007 (2016)]
}

P. S. Pasquini and O. L. G. Peres

(Received 29 March 2016; published 13 April 2016)

DOI: 10.1103/PhysRevD.93.079902

There is a typo on Eq. (11); it should read

$$
\left|g_{l}\right|^{2}=\sum_{\alpha=e, \mu, \tau}\left|g_{l \alpha}\right|^{2}+\left|h_{l \alpha}\right|^{2}
$$

which is in accordance with Eq. (3).

There is also a typo ${ }^{1}$ on Table I in our paper; the last line $\operatorname{Br}\left(K^{+} \rightarrow \mu^{+} \nu_{e} \nu \bar{\nu}\right)<6 \times 10^{-6}$ should be

$$
\operatorname{Br}\left(K^{+} \rightarrow \mu^{+} \nu_{\mu} \nu \bar{\nu}\right)<6 \times 10^{-6},
$$

so the table is

TABLE I. Reactions used in this work.

\begin{tabular}{lc}
\hline \hline Reaction & Reference \\
\hline$P \rightarrow l \bar{\nu}(\pi, K)$ & {$[17]$} \\
$P \rightarrow l \bar{\nu}\left(D, D_{s}, B\right)$ & {$[22-26]$} \\
$\pi^{+} \rightarrow e^{+} \nu_{H}$ & {$[27]$} \\
$K^{+} \rightarrow \mu^{+} \nu_{H}$ & {$[28]$} \\
$\operatorname{Br}\left(\pi^{+} \rightarrow e^{+} \nu_{e} \nu \bar{\nu}\right)<5 \times 10^{-6}$ & {$[17]$} \\
$\operatorname{Br}\left(K^{+} \rightarrow \mu^{+} \nu_{\mu} \nu \bar{\nu}\right)<6 \times 10^{-6}$ & {$[17]$} \\
\hline \hline
\end{tabular}

\footnotetext{
${ }^{1}$ We thank Yasaman Farzan for pointing this out.
} 